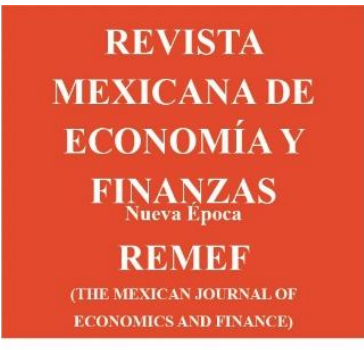

Revista Mexicana de Economía y Finanzas, Nueva Época

Volumen 16, Número 2, Abril - Junio 2021, pp. 1-28, e587

DOI: https://doi.org/10.21919/remef.v16i2.587

(Recibido: 24/agosto/2020, aceptado: 21/diciembre/2020, publicado: $28 /$ diciembre/2020)

\title{
Modelos de la estructura de plazos de las tasas de interés: Revisión, tendencias y perspectivas
}

\author{
Oldrich Alfons Vasicek ${ }^{1}$ - Vasicek Associates, U.S.A. \\ Francisco Venegas-Martínez ${ }^{2}$ - Instituto Politécnico Nacional, México
}

El trabajo proporciona una descripción general de los modelos de estructuras de plazos de las tasas de interés. Se trata de un planteamiento técnico de la teoría del comportamiento libre de arbitraje de tasas de interés de distintos vencimientos. Los modelos de tasa corta están ganando relevancia en la actualidad por su capacidad para describir y explicar la existencia de tasas de interés negativas como se ha observado en Europa y Asia. Las condiciones económicas actuales, en un entorno de incertidumbre generado por una recesión económica global, afectan el comportamiento de las tasas de interés, lo cual invita a realizar una revisión más cuidadosa de los factores que influyen en la dinámica de las mismas. Este artículo tiene como objetivo revisar las tendencias y perspectivas de los modelos de estructuras de plazos y destacar algunas áreas para futuras investigaciones.

Clasificación JEL: D50, E43.

Palabras clave: tasas de interés, estructuras de plazos, modelo de Vasicek.

\section{Models of the Term Structure of Interest Rates: Review, Trends and Perspectives}

The paper provides an overview of models of the term structure of interest rates. It is a technical exposition of the theory of arbitrage-free behavior of interest rates of different maturities. The short rate models acquire current relevance for their ability to describe and explain the existence of negative interest rates, as they have been observed in Europe and Asia. Current economic conditions, in an environment of uncertainty generated by a global economic recession, affect the behavior of interest rates, which invites a more detailed review of the factors that influence in their dynamics. This article aims to review the trends and perspectives of models of the term structure and highlights some areas for future research.

JEL classification: D50, E43.

Keywords: interest rates, term structure, Vasicek model

\footnotetext{
${ }^{1}$ Email. oldrich.vasicek@gmail.com

${ }^{2}$ Autor de correspondencia. Email. fvenegas1111@yahoo.com.mx

*Sin fuente de financiamiento para el desarrollo de la investigación.
} 


\section{Introducción}

Este trabajo proporciona una descripción general de los modelos de la estructura de plazos de las tasas de interés. Es una exposición técnica de la teoría del comportamiento libre de arbitraje de las tasas de interés de diferentes vencimientos. Los modelos están ganando relevancia en la actualidad por su capacidad para describir y explicar, a través de parámetros, la existencia de tasas de interés negativas, como se ha observado en Europa y Asia. Las condiciones económicas actuales, en un entorno de incertidumbre generado por una recesión económica global, afectan el comportamiento de las tasas de interés. El objetivo de la presente investigación consiste en revisar las tendencias y perspectivas de los modelos de la estructura de plazos de la tasa de interés y destaca algunas áreas para futuras investigaciones.

El trabajo está organizado de la siguiente manera: la sección 2 proporciona información detallada sobre la evolución de la teoría y los modelos de estructura de plazos que se han propuesto en la literatura; la sección 3 presenta un análisis a fondo del modelo de Heath, Jarrow y Morton (1992), el cual es un marco de referencia en la teoría de estructuras de plazo; la sección 4 establece algunas propuestas para futuras investigaciones; finalmente, la sección 5 proporciona las conclusiones

\section{Teoría de estructuras de plazo y curvas de rendimiento}

Las tasas de interés son una función del tiempo y del plazo (tiempo que falta para el vencimiento). En función del tiempo, las tasas se comportan como procesos estocásticos. En función del plazo, las tasas de interés en una fecha de referencia determinada constituyen la estructura de plazos, también llamada curva de rendimiento. Los modelos de estructura de plazos describen el comportamiento en el tiempo de las tasas de interés para diferentes plazos o vencimientos como un proceso estocástico conjunto

Los modelos de estructura de plazos son una herramienta necesaria para la valuación y la gestión de riesgos de los activos contingentes, o productos derivados, de tasas de interés, es decir, contratos cuyos pagos dependen de los valores futuros de las tasas de interés, como bonos callable (rescatables, derecho por parte del emisor de recomprar el bono al tenedor), bonos putable (derecho por parte del tenedor de devolver el bono al emisor), swaps, swaptions (el tenedor tiene el derecho de entrar a un swap), techos y pisos, etc. Para determinar el valor actual de un bono es necesario conocer el comportamiento posterior de las tasas de interés, lo mismo ocurre con todos los títulos de deuda sujetos a un pago anticipado, como las hipotecas con opciones de refinanciamiento

La aceptación y aplicación inmediata de los modelos de estructura de plazos en la práctica bancaria y de inversión se debió a que existen pocos instrumentos financieros cuyo valor no depende en ningún grado de las tasas de interés futuras. Incluso las opciones sobre acciones, de compra o venta, dependen de la dinámica futura de las tasas de interés. Asimismo, los modelos de tasas de interés se requieren en la valuación de las empresas y sus pasivos. Por último, los modelos de 
estructura de plazos son necesarios para la medición, gestión y cobertura del riesgo de tasa de

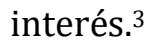

Las tasas de interés de diferentes vencimientos se comportan como un proceso estocástico conjunto. Sin embargo, no todos los procesos conjuntos pueden describir el comportamiento de las tasas de interés en un mercado eficiente. Por ejemplo, suponga que un modelo de estructura de plazos postula que las tasas de interés de todos los vencimientos cambian en el tiempo en cantidades iguales, es decir, las curvas de rendimiento se mueven mediante desplazamientos paralelos (lo que, empíricamente, parece ser una aproximación razonable de primer orden). Se puede demostrar que en este caso el rendimiento de un portafolio que consta de un bono largo (de plazo mayor a un año) y un bono corto (de plazo menor a un año) supera siempre a un bono a medio plazo con la misma duración de Macaulay (1983). No obstante, en un mercado eficiente, la oferta y la demanda reducirán el precio del bono de vencimiento medio y subirían los precios de los bonos largos y cortos. Equivalentemente, el rendimiento del bono medio aumenta y los rendimientos de los bonos largos y cortos disminuyen. Es decir, las curvas de rendimiento no permanecerán paralelas. En consecuencia, en el modelo de desplazamientos paralelos no es posible describir la dinámica de las tasas de interés.

Para que no existan oportunidades de arbitraje libres de riesgo, el proceso conjunto del comportamiento de las tasas de interés debe satisfacer algunas condiciones. Determinar estas condiciones y encontrar procesos que las satisfagan ha sido el propósito de la teoría de estructuras de plazos de la tasa de interés. Así, los modelos de estructura de plazos son aplicaciones específicas de la teoría de la estructura de plazos.

El proceso estocástico conjunto es conducido por una serie de fuentes de incertidumbre. Para los procesos continuos, las fuentes de incertidumbre a menudo se especifican como movimientos brownianos o procesos de Wiener. Si la evolución de la curva de rendimiento se puede representar mediante variables de estado markovianas, estas variables se llaman, usualmente, factores.

\subsection{Algunas definiciones necesarias}

Todas las tasas son anualizadas y continuamente capitalizables. Un préstamo a plazo $T$ a una tasa fija continuamente capitalizable $r$ paga al vencimiento el monto $\exp (r T)$. Una tasa $R_{n}$ que se compone con frecuencia anual $n$ (por ejemplo, si $n=12, R_{n}$ es la tasa con composición mensual) se relaciona con la tasa continuamente capitalizable $r$ mediante

$$
1+R_{n} / n=e^{r / n}
$$

Sea $B(t, T)$ el precio a descuento de un bono cupón cero, al tiempo $t$, que paga una tasa de interés libre de riesgo de incumplimiento que vence en el tiempo $T$ y que paga al vencimiento una unidad monetaria. El rendimiento al vencimiento $R(t, S)$ en el momento $t$ con plazo $S=T-t$ se define como la tasa de rendimiento continuamente capitalizable del bono, y está dado por:

\footnotetext{
${ }^{3}$ Véanse, por ejemplo, Venegas-Martínez (2002) y (2003), así como Venegas-Martínez y González-Aréchiga (2002).
} 


$$
R(t, S)=-\frac{1}{S} \log B(t, t+S)
$$

La tasa de interés instantánea recibe el nombre de tasa corta y está dada por:

$$
r(t)=\lim _{S \rightarrow 0} R(t, S)
$$

Un activo que acumula intereses a una tasa corta se denomina cuenta de mercado de dinero:

$$
M(t)=\exp \left(\int_{0}^{t} r(\tau) \mathrm{d} \tau\right)
$$

Las tasas forward $f(t, T)$ se definen mediante la ecuación

$$
B(t, T)=\exp \left(-\int_{t}^{T} f(t, \tau) \mathrm{d} \tau\right)
$$

o equivalentemente,

$$
f(t, T)=-\frac{\partial}{\partial T} \log B(t, T)
$$

Las tasas forward son las tasas de rendimiento marginales por realizar una inversión en bonos para un instante adicional. La tasa forward para la fecha actual está dada por

$$
f(t, t)=r(t)
$$

La mayoría de los bonos pagan cupones durante su vigencia. Un bono que paga cupones (que realiza pagos intermedios durante el plazo) es sólo un paquete de bonos a descuento, uno por cada cupón o pago de capital.

\subsection{Modelos de un factor}

La teoría general de los modelos de estructura de plazos de un factor fue propuesta por Vasicek (1977). En esta teoría se supone que:

(A1) La tasa corta sigue un proceso continuo Markoviano. 
(A2) El precio $B(t, T)$ de un bono se determina mediante la valuación en el tiempo $t$ del segmento $\{r(\tau), t \leq \tau \leq T\}$ del proceso de tasa corta durante el plazo del bono.

(A3) El mercado es eficiente, es decir, la información está disponible para todos los inversionistas simultáneamente y cada inversionista actúa racionalmente (prefiere más riqueza a menos y usa toda la información disponible) y no hay costos de transacción.

El supuesto (A3) implica que los inversionistas tienen expectativas homogéneas y que no es posible un arbitraje rentable sin riesgo. Por el supuesto (A1), la dinámica de la tasa corta en un intervalo [t,T], $t \leq T$, dados sus valores antes del tiempo $t$, depende sólo del valor actual $r(t)$. El supuesto (A2) implica entonces que el precio $B(t, T)$ es una función de $r=r(t)$. Por tanto, el valor de la tasa corta es la única variable de estado para toda la estructura de plazo. Suponga que la dinámica de la tasa corta está dada por una ecuación diferencial estocástica de la forma:

$$
\mathrm{d} r(t)=\zeta(r, t) \mathrm{d} t+\varphi(r, t) \mathrm{d} W(t)
$$

donde $W(t)$ es un proceso de Wiener (o movimiento browniano estándar). Denote ahora la media y la varianza de la tasa de rendimiento instantánea del bono con precio $B(t, T)$ por $\mu(t, T)$ y $\sigma^{2}(t, T)$, respectivamente, de tal manera que

$$
\frac{\mathrm{d} B(t, T)}{B(t, T)}=\mu(t, T) \mathrm{d} t-\sigma(t, T) \mathrm{d} W(t)
$$

El signo negativo en la volatilidad $\sigma(t, T)$ no conlleva ningún problema ya que $W(t)$ y $-W(t)$ tienen la misma distribución. Considere ahora un inversionista que al tiempo $t$ emite una cantidad $w_{1}$ de un bono con fecha de vencimiento $T_{1}$, y simultáneamente compra una cantidad $w_{2}$ de un bono con fecha de vencimiento $T_{2}$. Suponga que las cantidades $w_{1} \mathrm{y} w_{2}$ se eligen para que sean proporcionales a $\sigma\left(t, T_{2}\right)$ y $\sigma\left(t, T_{1}\right)$, respectivamente. Por lo tanto, la posición es instantáneamente libre de riesgos y debería pagar la tasa de rendimiento corta $r(t)$. De ello se deduce que la razón $(\mu(t, T)-$ $r(t)) / \sigma(t, T)$ es independiente de $T$. Su valor común $\lambda(t)$ se denomina precio de mercado del riesgo, ya que especifica el aumento en la tasa de rendimiento esperada de un bono por unidad adicional de riesgo. De esta manera,

$$
\mu(t, T)=r(t)+\lambda(t) \sigma(t, T)
$$

Al aplicar el lema de Itô al precio $B=B(t, T, r)$ y comparar el resultado con la ecuación (8) tomando en cuenta (9) se obtiene

$$
\frac{\partial B}{\partial t}+(\zeta+\varphi \lambda) \frac{\partial B}{\partial r}+\frac{1}{2} \varphi^{2} \frac{\partial^{2} B}{\partial r^{2}}-r B=0
$$


Esta es una ecuación diferencial parcial de segundo orden. El precio del bono está sujeto a la condición de frontera $B(T, T)=1$ (paga una unidad monetaria al vencimiento). La solución de (10) está dada por:

$$
B(t, T)=\mathrm{E}_{t} \exp \left(-\int_{t}^{T} r(\tau) \mathrm{d} \tau-\frac{1}{2} \int_{t}^{T} \lambda^{2}(\tau) \mathrm{d} \tau+\int_{t}^{T} \lambda(\tau) \mathrm{d} W(\tau)\right)
$$

Esta ecuación, denominada ecuación fundamental de determinación de precios de bonos (la ecuación de Vasicek) describe completamente la estructura de plazos y su comportamiento. Observe que la ecuación del precio de un bono se obtuvo inicialmente como la solución de una ecuación diferencial parcial de segundo orden bajo ciertos supuestos, pero es válida en general para cualquier modelo de estructura de plazos libre de arbitraje. La ecuación es válida incluso en el caso de múltiples factores o múltiples fuentes de riesgo si los productos de la ecuación se interpretan como productos escalares de vectores. Cada modelo de estructura de plazos es una aplicación directa de (11) o supone que (11) se cumple para los bonos y se usa para determinar el precio de los derivados de tasa de interés (como en el modelo de Heath, Jarrow y Morton).

\subsection{El modelo de Vasicek}

Vasicek (1977) proporciona un ejemplo de un modelo de estructura de plazos en el que la tasa corta sigue una caminata aleatoria con reversión a la media (el proceso de Ornstein-Uhlenbeck)

$$
\mathrm{d} r=\kappa(\theta-r) \mathrm{d} t+\varphi \mathrm{d} W
$$

y el precio de mercado del riesgo $\lambda(t, r)=\lambda$ es constante. El proceso de Ornstein-Uhlenbeck es un proceso de Markov con incrementos distribuidos normalmente y una distribución estacionaria. La tendencia instantánea $\kappa(\theta-r)$ representa una fuerza (o mecanismo) que lleva al proceso hacia su media de largo plazo $\theta$ con una magnitud proporcional a la desviación del proceso desde la media y la constante $\kappa$ representa la velocidad de ajuste. El elemento estocástico $\varphi d W$ hace que el proceso fluctúe alrededor de $\theta$ de forma aleatoria pero continua. La esperanza condicional y la varianza del proceso, dado el nivel actual, satisfacen

$$
\mathrm{E}_{t} r(T)=\theta+(r(t)-\theta) e^{-\kappa(T-t)}
$$

$$
\operatorname{Var}_{t} r(T)=\frac{\varphi^{2}}{2 \kappa}\left(1-e^{-2 \kappa(T-t)}\right)
$$


respectivamente. De esta manera,

$$
B(t, T)=\exp \left(D(t, T)(R(\infty)-r)-(T-t) R(\infty)-\frac{\varphi^{2}}{4 \kappa} D^{2}(t, T)\right)
$$

donde

$$
D(t, T)=\frac{1}{\kappa}\left(1-e^{-\kappa(T-t)}\right)
$$

$\mathrm{y}$

$$
R(\infty)=\theta+\lambda \varphi / \kappa-\frac{1}{2} \varphi^{2} / \kappa^{2}
$$

La función $D(t, T)$ describe la exposición del precio del bono al factor estocástico $r(t)$. Suponga que la media $\mu(t, T)$ y la desviación estándar $\sigma(t, T)$ de la tasa de rendimiento instantánea de un bono que vence al tiempo $T$ tienen la forma:

$$
\begin{aligned}
& \mu(t, T)=r(t)+\lambda \varphi D(t, T)), \\
& \sigma(t, T)=\varphi D(t, T) .
\end{aligned}
$$

De esta manera, cuanto más largo sea el plazo del bono, mayor será la varianza de la tasa de rendimiento instantánea. En este caso, el rendimiento esperado en exceso de la tasa spot (de mercado) es proporcional a la desviación estándar. Para un vencimiento muy largo (es decir, $T \rightarrow \infty$ ), la media y la desviación estándar se aproximan a los límites

$$
\begin{aligned}
& \mu(t, \infty)=r(t)+\lambda \varphi / \kappa, \\
& \sigma(t, \infty)=\varphi / \kappa .
\end{aligned}
$$

estructura de plazos de las tasas de interés se calcula ahora a partir de (2) y (15) como

$$
R(t, S)=R(\infty)+(r(t)-R(\infty)) \frac{1}{S} D(t, t+S)+\frac{\varphi^{2}}{4 \kappa S} D^{2}(t, t+S) .
$$

Es importante tener en cuenta que el rendimiento de un bono muy largo, con $S \rightarrow \infty$, es $R(\infty)$, lo que explica la notación en (17). Las curvas de rendimiento dadas por (20) comienzan en el nivel actual $r(t)$ de la tasa spot para $S=0$ y se acercan a una asíntota común $R(\infty)$ cuando $S \rightarrow \infty$. Dependiendo del valor de $r(t)$, la curva de rendimiento puede ser monótona creciente, una curva jorobada o monótona decreciente y las tasas de interés son gaussianas. La ventaja del modelo Vasicek es su manejabilidad. Otra ventaja importante de este modelo es que en la actualidad existen tasas de interés negativas y este modelo resulta ser una herramienta útil para la descripción y explicación de este fenómeno. 


\subsection{Ejemplos de modelos de estructura de plazos}

En la literatura se han propuesto varios casos específicos de modelos de estructura de plazos. Por ejemplo, Cox, Ingersoll y Ross (1985) obtienen un modelo en el que la tasa corta $r=r(t)$ sigue un proceso de la forma

$$
\mathrm{d} r=\kappa(\theta-r) \mathrm{d} t+\varphi \sqrt{r} \mathrm{~d} W
$$

Para este modelo, el precio de mercado del riesgo está dado por $\lambda(t, r)=\eta \sqrt{r}$. En este caso, los precios de los bonos también se pueden expresar explícitamente mediante:

$$
B(t, T)=A(t, T) \exp (-D(t, T) r(t))
$$

La cantidad $D(t, T)$ que mide el grado de exposición de los precios de los bonos al factor estocástico $r(t)$, está dada por

$$
D(t, T)=\frac{1-e^{-\gamma(T-t)}}{\gamma+\frac{1}{2}(\kappa-\varphi \eta-\gamma)\left(1-e^{-\gamma(T-t)}\right)}
$$

Donde

$$
\gamma=\left(2 \varphi^{2}+(\kappa-\varphi \eta)^{2}\right)^{1 / 2}
$$

En este caso, las tasas de interés son siempre no negativas (conducidas por una Ji cuadrada no central). El modelo de Cox-Ingersoll-Ross (CIR) parte de un modelo macroeconómico de equilibrio general, lo cual contrasta con la mayoría de modelos que se obtienen mediante argumentos sin arbitraje. Observe que en su investigación de 1985, Cox, Ingersoll, Ross usan la notación $\lambda=-\varphi \eta$, lo que da una impresión errónea sobre el signo del precio de mercado del riesgo. Para una prima de riesgo de bonos positiva $\mathrm{E}[\mathrm{d} B / B]-r=\eta \sqrt{r} \mathrm{E}[\mathrm{d} W \mathrm{~d} B / B]$, la cantidad $\lambda$ del modelo CIR debe ser negativa.

Es por lo anterior necesario imponer algunas restricciones a los coeficientes del modelo para evitar comportamientos singulares. Por ejemplo, el proceso $r=r(t)$ tendrá reversión negativa a la media negativa (repulsión a la media) bajo la medida de martingala si $\kappa-\varphi \eta<0$. Cuando $\kappa-\varphi \eta<-$ $\varphi \sqrt{2}$, el balance esperado en una cuenta de mercado de dinero será infinito en un tiempo finito.

Hull y White (1990) ampliaron los modelos de Vasicek y CIR permitiendo que los parámetros en (12) y (21), así como el precio de mercado del riesgo, dependan del tiempo. Esto tiene la ventaja de que el modelo se puede hacer consistente con los datos iniciales. Por ejemplo, haciendo $\theta$ función del tiempo, se puede hacer que el modelo se ajuste exactamente a la estructura de plazos inicial de las tasas de interés (lo que no es posible con modelos homogéneos en el tiempo). Del mismo modo, 
hacer la volatilidad $\varphi$ una función del tiempo permite calibrar el modelo a la estructura de plazos de las volatilidades en swaptions. Hull y White ofrecen soluciones de forma cerrada para los precios de los bonos que ellos llaman los modelos extendidos de Vasicek y CIR. Estos casos pertenecen a la clase de modelos que Duffie y Kan (1994) denominan modelos de estructura de plazos afines, en los que los precios de los bonos tienen la forma dada en (12) ó (21).

Por otro lado, Black, Derman y Toy (1990) y Black y Karasinski (1991) desarrollan un modelo que parte de la siguiente ecuación diferencial estocástica:

$$
\mathrm{d} \log r=\kappa(t)(\log \theta(t)-\log r) \mathrm{d} t+\varphi(t) \mathrm{d} W
$$

En este caso, los precios de los bonos no se pueden obtener en fórmulas cerradas, pero se pueden aproximar numéricamente. Se observa también que las tasas de interés son lognormales.

A diferencia de otros modelos, los lognormales no son consistentes con el equilibrio del mercado. Es decir, no existe equilibrio económico en la que estos modelos puedan describir las tasas de interés; esto se puede probar formalmente. Un indicio de los problemas que tienen los modelos lognormales es que el balance futuro esperado (iincluso en un instante!) en la cuenta del mercado de dinero es infinito,

$$
E \frac{M(T)}{M(t)}=\infty, \quad t<T
$$

Es importante destacar que los modelos lognormales pueden producir precios futuros infinitos.

\subsection{Clasificación de los modelos de estructura de plazos}

Para clasificar los numerosos modelos de estructura de plazos propuestos en la literatura es necesario tener en cuenta varios aspectos:

1. Número de fuentes de riesgo / número de factores:

Modelos de un factor. El factor suele ser la tasa corta

Dos factores (Brennan-Schwartz, 1979, Longstaff-Schwartz, 1992)

Múltiples factores (Langetieg 1980)

Modelos sin factores (Heath-Jarrow-Morton, 1990, 1992)

Los factores son variables de estado que contienen toda la información disponible. Los factores son también procesos markovianos de tal modo que las tasas y los precios son funciones únicamente de los factores. Los modelos de factores se construyen de la siguiente manera:

Especificar factores estocásticos

Describir el precio del riesgo. 
Resolver la ecuación diferencial parcial para los precios de los bonos. ${ }^{4}$

\section{La naturaleza del proceso.}

Reversión a la media vs. caminata aleatoria

Procesos normales vs. lognormales frente a procesos de raíz cuadrada.

\section{Estructura de plazos inicial:}

Curva de rendimiento implícita (modelos normativos). Estos modelos prescriben una familia de curvas que puede alcanzarse mediante la estructura de plazos (por ejemplo, Vasicek, CoxIngersoll-Ross, Longstaff-Schwartz). Se supone que la curva de rendimiento inicial pertenece a esa familia. La desventaja obvia es que el modelo no coincide exactamente con el precio actual de los bonos. La diferencia entre el rendimiento real e implícito se atribuye a la determinación de precios incorrectos o a características específicas de los bonos, como la liquidez. La ventaja es que las curvas de rendimiento futuras implícitas son realistas.

Curva de rendimiento ajustada (modelos descriptivos). Estos modelos toman la curva de rendimiento inicial como dada (por ejemplo, Hull-White, Ho-Lee, Heath-Jarrow-Morton) y se ajustan exactamente al precio actual de los bonos. La desventaja es que el usuario sabe que mañana estaría cambiando cantidades (la familia de posibles curvas de rendimiento) que ahora él considera deterministas. Por supuesto, conviene actualizar los parámetros con la llegada de nueva información, pero no para violar sistemáticamente los supuestos.

Los modelos multifactoriales que son modelos normativos de coeficientes constantes con un número suficiente de factores (por ejemplo, rendimientos de referencia). Dichos modelos (por ejemplo, Duffie y Kan, 1994, y Vasicek, 2020), aunque computacionalmente son complejos, tienen las ventajas de los modelos normativos y descriptivos sin las desventajas de ninguno de los dos.

Vale la pena hacer un listado, en el Cuadro 1, de algunos de los modelos de un factor que fueron motivados por la propuesta inicial de Vasicek. La lista no pretende ser exhaustiva, y en ella se muestra el desarrollo de los modelos de estructuras de plazo.

Cuadro 1. Algunos modelos de un factor y sus propiedades

\begin{tabular}{|l|c|l|l|c|c|}
\hline \multicolumn{1}{|c|}{ Modelo } & Vasicek & Hull/White & Cox/Ingersoll/Ross & Black/Derman/Toy & Ho/Lee \\
\hline $\begin{array}{l}\text { Reversión a la } \\
\text { media }\end{array}$ & Lineal & Lineal & Lineal & No lineal & Ninguna \\
\hline Volatilidad & $\varphi$ & $\varphi$ & $\varphi \sqrt{r}$ & $\varphi r$ & $\varphi$ \\
\hline $\begin{array}{l}\text { Parámetros } \\
\text { constantes }\end{array}$ & Sí & No & Sí & No & No \\
\hline
\end{tabular}

\footnotetext{
${ }^{4}$ La cobertura del riesgo de tasa de interés es posible en los modelos de factores.
} 


\begin{tabular}{|l|c|c|c|c|c|}
\hline $\begin{array}{l}\text { Tasas de } \\
\text { interés no } \\
\text { negativas }\end{array}$ & No & No & Sí & Sí \\
\hline $\begin{array}{l}\text { Distribución } \\
\text { de la tasa de } \\
\text { interés }\end{array}$ & Normal & Normal & $\begin{array}{c}\text { Chi (Ji) cuadrada no } \\
\text { central }\end{array}$ & Lognormal & Normal \\
\hline $\begin{array}{l}\text { Solución en } \\
\text { forma cerrada }\end{array}$ & Sí & Sí & Sí & No & Sí \\
\hline
\end{tabular}

Fuente: Elaboración de los autores

Claramente, todos los modelos descriptivos mencionados en el Cuadro 1 tienen inconvenientes. La delimitación más obvia es, por supuesto, el supuesto de normalidad; aunque este supuesto, usualmente, permite encontrar soluciones cerradas. Asimismo, el supuesto de volatilidad constante es otro problema. También, la dinámica de la tasas de interés responde a múltiples factores y diversas fuentes de incertidumbre y los modelos anteriores sólo consideran un factor. Por último, es de resaltarse que lo que antes era una debilidad en varios de los modelos, específicamente, la generación de tasas cortas negativas, en la actualidad es una ventaja ya permiten describir y explicar la existencia de tasas de interés negativas que se han observado en varias regiones en el mundo.

\subsection{Precios de contratos contingentes (productos derivados)}

Una de las aplicaciones de los modelos de estructura de plazos es la determinación de precios de los contratos contingentes de tasas de interés (productos derivados de tasa de interés). Esto se podría abordar de varias formas. Para los modelos de un factor, se puede demostrar, mediante un argumento de arbitraje similar a los anteriormente expuestos para los bonos, que el precio $P(t)$ de cualquier derivado de tasa de interés satisface también la ecuación diferencial parcial (9). La valuación del derivado se obtiene resolviendo dicha ecuación sujeta a condiciones de frontera que describen los pagos de los productos derivados.

Por ejemplo, las condiciones de frontera para un bono cupón cero con vencimiento en el tiempo $T_{M}$ con valor nominal $F$, llamable después del tiempo $T_{C}$ al precio de una opción de compra $C$ son:

$$
P\left(T_{M}, r\right)=F, \quad P(t, r) \leq C \text { for } T_{C} \leq t \leq T_{M} .
$$

Si no se puede obtener una solución de forma cerrada, entonces la solución se puede aproximar numéricamente mediante un árbol o en una red de diferencias finitas. Un método más general es darse cuenta de que dicha solución tiene la forma

$$
P(t)=\mathrm{E}_{t} P(T) \exp \left(-\int_{t}^{T} r(\tau) \mathrm{d} \tau-\frac{1}{2} \int_{t}^{T} \lambda^{2}(\tau) \mathrm{d} \tau+\int_{t}^{T} \lambda(\tau) \mathrm{d} W(\tau)\right)
$$


Esta ecuación es válida incluso en los casos en los que no hay variables de estado markovianas. Sin embargo, calcular la esperanza en la ecuación anterior puede ser más difícil que resolver una ecuación diferencial parcial.

\subsection{Prima de liquidez}

La diferencia entre la tasa forward y la tasa al contado esperada se ha denominado tradicionalmente prima de liquidez. Sea

$$
\varphi(t, T)=\frac{\partial \sigma(t, T)}{\partial T}
$$

la volatilidad de la tasa de interés forward $f(t, T)$. La prima de liquidez (o prima de plazo, como debería llamarse) $\pi(t, T)$ viene dada por

$$
\pi(t, T)=f(t, T)-\mathrm{E}_{t} r(T)=\mathrm{E}_{t} \int_{t}^{T} \varphi(\tau, T) \lambda(\tau) \mathrm{d} \tau-\mathrm{E}_{t} \int_{t}^{T} \varphi(\tau, T) \sigma(\tau, T) \mathrm{d} \tau
$$

La prima de liquidez en una estructura de plazo de tasas de interés tiene dos componentes: el primer componente está determinado por el precio de mercado del riesgo, el cual es igual al agregado esperado del precio de mercado del riesgo durante el plazo de la tasa forward, ponderado por la volatilidad de la tasa forward. El segundo componente es igual al valor negativo del agregado esperado de la volatilidad del precio del bono multiplicado por la volatilidad de la tasa forward sobre el plazo de la tasa forward. Este último componente está presente incluso si el precio de mercado del riesgo es cero; surge como resultado de la relación no lineal entre precios y tasas.

\subsection{La medida de la martingala}

La teoría moderna de valuación de derivados (véase Harrison y Kreps, 1979) introduce un cambio de medida de probabilidad como herramienta básica de determinación de precios. A saber, existe una medida de probabilidad equivalente, $\mathbf{P}^{*}$, denominada medida de martingala equivalente, $\mathbf{o}$ (incorrectamente) medida neutral al riesgo, de modo que el valor $P(t)$ de cualquier activo expresado en unidades de la cuenta del mercado de dinero $M(t)$ sigue una martingala bajo esa medida,

$$
\frac{P(t)}{M(t)}=\mathrm{E}_{t}^{*} \frac{P(T)}{M(T)} .
$$




$$
P(t)=\mathrm{E}_{t}^{*} P(T) \exp \left(-\int_{t}^{T} r(\tau) \mathrm{d} \tau\right)
$$

en cualquier intervalo $(t, T)$ en donde el activo $P(t)$ no realiza pagos. El proceso

$$
W^{*}(t)=W(t)-\int_{0}^{t} \lambda(\tau) \mathrm{d} \tau
$$

es un proceso de Wiener bajo la medida de probabilidad neutral al riesgo $\mathbf{P}^{*}$. Para cada activo $P(t)$, se sigue que

$$
\left.\frac{\mathrm{d} P}{P}=\left(r+\sigma_{P} \lambda\right) \mathrm{d} t-\sigma_{P} \mathrm{~d} W\right)=r \mathrm{~d} t-\sigma_{P} \mathrm{~d} W^{*}
$$

El precio del riesgo bajo la medida martingala es cero. La teoría establece que los activos financieros se valúan como el valor esperado de sus flujos de efectivo futuros descontados al presente con el promedio de la tasa corta en el intervalo $(t, T)$. La esperanza se toma con respecto a la medida martingala bajo la cual la tasa de rendimiento esperada de todos los activos es la tasa corta. Los precios de los bonos están dados por

$$
B(t, T)=\mathrm{E}_{t}^{*}\left[\exp \left\{-\int_{t}^{T} r(\tau) \mathrm{d} \tau\right\} \mid r(t)\right]
$$

Por otro lado, la derivada Radon-Nikodym de la nueva medida con respecto a la medida actual es

$$
\frac{\mathrm{d} \mathbf{P}^{*}}{\mathrm{~d} \mathbf{P}}=\exp \left(-\frac{1}{2} \int_{t}^{T_{\max }} \lambda^{2}(\tau) \mathrm{d} \tau+\int_{t}^{T_{\max }} \lambda(\tau) \mathrm{d} W(\tau)\right)
$$

Ya que

$$
\mathrm{E}^{*} X=\mathrm{E} X \frac{\mathrm{d} \mathbf{P}^{*}}{\mathrm{~d} \mathbf{P}}
$$

para cualquier variable $X$, al aplicar las ecuaciones (34) y (35) a un bono se produce la ecuación fundamental de valuación de los bonos (11). 


\subsection{El modelo de Heath-Jarrow-Morton}

El modelo de Heath, Jarrow y Morton (1992) es en realidad más que un modelo, es un marco de referencia. Si bien el modelo es coherente con la ecuación fundamental de determinación de precios de los bonos (11), éste permite determinar el precio de los derivados de tasas de interés sin conocer el precio de mercado del riesgo $\lambda(t)$. El precio del riesgo se obtiene implícitamente de los precios actuales de los bonos. Este enfoque fue propuesto en esencia por Ho y Lee (1986) y luego formalizado por Heath, Jarrow y Morton. El conocimiento de la estructura de plazo inicial $f(0, T), T \geq 0$ y de las volatilidades de las tasas forward es suficiente para determinar el precio de los contratos dependientes de la tasa de interés. El modelo supone que se conocen los precios actuales de los bonos de todos los vencimientos y utiliza la curva de rendimiento inicial para determinar el precio de los derivados de tasa de interés. Al escribir la dinámica de las tasas forward directamente en términos del proceso $W^{*}(t)$, es posible determinar el precio de los contratos contingentes de tasas de interés sin conocer explícitamente el precio de mercado del riesgo; las volatilidades de las tasas forward son un insumo del modelo. El modelo se ajusta a los precios actuales de los bonos y genera una dinámica de las tasas forward mediante:

$$
f(t, T)-f(0, T)=\int_{0}^{t} \varphi(\tau, T) \sigma(\tau, T) \mathrm{d} \tau+\int_{0}^{t} \varphi(\tau, T) \mathrm{d} W^{*}(\tau)
$$

donde $\varphi(t, T)$ es la volatilidad de la tasa forward $f(t, T)$ y

$$
\sigma(\tau, T)=\int_{\tau}^{T} \varphi(\tau, s) \mathrm{d} s
$$

es la volatilidad del bono. Si $W^{*}, \varphi$ y $\sigma$ son vectores, sus productos se interpretan como productos internos.

La relevancia de la ecuación (37) es que determina el valor esperado bajo $\mathbf{P}^{*}$ de las tasas forward, infiriéndolo del precio actual y, por lo tanto, conteniendo implícitamente la prima de riesgo. Los pagos de cualquier contrato contingente de tasa de interés se pueden expresar en términos de tasas forward futuras. El contrato es valuado como el valor esperado de sus pagos descontados según la medida de martingala para la cual $W^{*}(t)$ es un proceso de Wiener.

A continuación se proporciona una derivación de tres líneas del modelo de Heath-JarrowMorton (1992); en la sección 3 haremos una deducción detallada. Con base en las ecuaciones (8), (9) y (32) los precios de los bonos están sujetos a

$$
\frac{\mathrm{d} B(\tau, T)}{B(\tau, T)}=r(\tau) \mathrm{d} \tau-\sigma(\tau, T) \mathrm{d} W^{*}(\tau)
$$

Después de integrar la ecuación anterior con respecto de $\tau$ de 0 a $t$, se tiene que 


$$
\log B(t, T)-\log B(0, T)=\int_{0}^{t} r(\tau) \mathrm{d} \tau-\frac{1}{2} \int_{0}^{t} \sigma^{2}(\tau, T) \mathrm{d} \tau+\int_{0}^{t} \sigma(\tau, T) \mathrm{d} W^{*}(\tau)
$$

y diferenciando la ecuación anterior con respecto a $T$, tomando en cuenta (6), se produce la ecuación (37).

\subsection{La curva de rendimiento}

Un gráfico de los rendimientos de los bonos en una fecha determinada frente al vencimiento se denomina curva de rendimiento. Dado que las cotizaciones de rendimiento suelen estar disponibles sólo para vencimientos seleccionados (los denominados rendimientos de referencia), ha sido necesario ajustar una curva suave a los datos discretos. Se ha realizado un esfuerzo sustancial en la interpolación de la curva de rendimiento (véanse, por ejemplo, McCulloch 1971; Vasicek y Fong 1982; Nelson y Siegel 1987; Adams y van Deventer 1994). Aunque estos métodos funcionan razonablemente en la práctica, las interpolaciones resultantes no corresponden a ningún modelo de estructura de plazos libre de arbitraje. No existe una estructura de plazos de equilibrio de tasas de interés bajo la cual las curvas de rendimiento formen splines, ya sean cúbicos, cuadráticos o exponenciales. Vasicek (2020) propone una interpolación de la curva de rendimiento compatible con un modelo libre de arbitraje de la estructura de plazos de la tasa de interés. El modelo es un modelo de factor de rendimiento gaussiano homogéneo en el tiempo multivariante. Para que el método de interpolación dé como resultado curvas de rendimiento estables, se introduce la interpolación de máxima estabilidad como aquella que minimiza la integral de la variación de rendimiento a lo largo del plazo.

\section{Revisitando el modelo de Heath-Jarrow-Morton}

En esta sección se presenta, detalladamente, la metodología desarrollada por David Heath, Robert Jarrow y Andrew Morton (HJM) en su artículo "Bond Pricing and the Term Structure of Interest Rates: A New Methodology for Contingent Claims Valuation", publicado en 1992 en Econometrica, en el cual se generan curvas de rendimiento con base en la tasa forward actual (a todos los vencimientos) y una curva de rendimiento inicial. Asimismo, se desarrollan varios ejemplos de la metodología HJM.

Para estimar los precios de un bono cupón cero a diferentes vencimientos, el modelo de Heath, Jarrow y Morton comienza con una especificación exógena de la dinámica estocástica de la tasa forward y, posteriormente, determina endógenamente, en un mundo neutral al riesgo, la dinámica estocástica de un bono cupón cero.

La metodología HJM es similar a la de Ho y Lee (1986) y Hull y White (1990) en varios sentidos. En primer lugar, se requiere una curva de rendimiento inicial, la cual es proporcionada por el mercado en una fecha anterior. Asimismo, la tendencia de la tasa forward instantánea se calibra de tal forma que el premio al riesgo estandarizado por volatilidad es cero. Las diferencias con Ho y Lee (1986) y Hull y White (1990) son, básicamente: 1) el proceso de valuación en HJM se inicia con una especificación exógena de la dinámica estocástica de la tasa forward, 2) la hipótesis de expectativas en HJM para valuar un bono es que el nominal se descuenta con el promedio de la tasa forward durante la vigencia del instrumento, razón por lo que el precio del bono es una variable aleatoria y 
3) la calibración en HJM es un procedimiento implícito en la metodología y no requiere argumentos de ajuste como en el caso de Ho y Lee (1986) y Hull y White (1990). En virtud de que, bajo la metodología HJM, el precio de un bono es una variable aleatoria, el método Monte Carlo es una herramienta muy útil en la práctica. Una ventaja de la metodología HJM que debe destacarse es que puede ser extendida a varios factores de riesgo; por ejemplo, factores de corto y largo plazo. No obstante, una limitación de la metodología HJM es que se pueden producir tasas forward negativas con probabilidad positiva.

Vale la pena mencionar que cuando se parte de una especificación exógena para la dinámica estocástica de la tasa corta, la hipótesis de expectativas para valuar un bono es que, primero, el nominal se descuenta con el promedio de la tasa corta durante la vigencia del título y, posteriormente, se toma el valor esperado condicional en la información disponible en la fecha de colocación. A partir del Teorema de Feynman-Kac se tiene que esta es la única hipótesis de expectativas que es congruente con el enfoque de ecuaciones diferenciales parciales. De esta manera, la metodología HJM no es compatible con dicho enfoque.

\subsection{Especificación exógena de la tasa forward instantánea}

Considere un movimiento Browniano estándar $(W(t))_{t \in[0, T]}$ definido sobre un espacio fijo de probabilidad con su filtración aumentada $\left(\Omega, F,\left(F_{t}\right)_{t \in[0, T]}, \mathbf{P}\right)$. Por simplicidad, en lo que sigue se escribirá $W(t)=W_{t}$. En la metodología HJM se supone que la dinámica de la tasa forward, $f(t, T)$, se especifica exógenamente por la siguiente ecuación diferencial estocástica ${ }^{5}$

$$
\mathrm{d} f(t, T)=\alpha(t, T) \mathrm{dt}+\varphi(t, T) \mathrm{d} W_{t}
$$

en donde, de acuerdo con (38), las funciones $\alpha(t, T)$ y $\varphi(t, T)$ satisfacen, casi seguramente con respecto de $\mathbf{P}$, las siguientes propiedades:

$$
\int_{0}^{T}\left|\frac{\partial^{k}}{\partial T^{k}} \alpha(s, T)\right| \mathrm{d} s<\infty \quad \text { y } \quad \int_{0}^{T}\left|\frac{\partial^{k}}{\partial T^{k}} \varphi(s, T)\right|^{2} \mathrm{~d} s<\infty
$$

para $k=0$, 1 . Como siempre, $\partial^{0} \alpha(s, T) / \partial T^{0} \equiv \alpha(s, T)$ y $\partial^{0} \varphi(s, T) / \partial T^{0} \equiv \varphi(s, T)$. Asimismo, se supone que el precio de un bono cupón cero está dado por

$$
B(t, T)=\exp \left\{-\int_{t}^{T} f(t, s) \mathrm{d} s\right\}
$$

lo cual define la tasa forward siempre; la integral anterior debe permanecer finita. Una de las tareas de la presente sección consiste en determinar endógenamente el proceso asociado al precio, $B(t, T)$,

${ }^{5}$ Véase, por ejemplo, Venegas-Martínez (2008). 
que haga consistentes los supuestos (41) y (42). La ecuación (41) puede considerar más de un factor de incertidumbre, por el momento el análisis subsecuente tomará en cuenta un solo factor.

\subsection{Dinámica estocástica de la tasa corta}

En esta sección se determina la ecuación diferencial estocástica que conduce a la tasa corta. Observe primero que a partir de (41) se obtiene

$$
f(t, T)=f(0, T)+\int_{0}^{t} \alpha(s, T) \mathrm{d} s+\int_{0}^{t} \varphi(s, T) \mathrm{d} W_{s}
$$

Por lo tanto, la tasa instantánea satisface

$$
r_{t}=f(t, t)=f(0, t)+\int_{0}^{t} \alpha(s, t) \mathrm{d} s+\int_{0}^{t} \varphi(s, t) \mathrm{d} W_{s}
$$

De esta manera,

$$
\mathrm{E}\left[r_{t} \mid F_{t}\right]=f(0, t)+\int_{0}^{t} \alpha(s, t) \mathrm{d} s
$$

$\mathrm{y}$

$$
\operatorname{Var}\left[r_{t} \mid F_{t}\right]=\int_{0}^{t} \varphi^{2}(s, t) \mathrm{d} s
$$

Asimismo, observe que la diferencial estocástica de la tasa corta está dada por:

$$
\mathrm{d} r_{t}=\frac{\partial f(0, t)}{\partial t} \mathrm{~d} t+\frac{\partial}{\partial t}\left(\int_{0}^{t} \alpha(s, t) \mathrm{d} s\right) \mathrm{d} t+\frac{\partial}{\partial t}\left(\int_{0}^{t} \varphi(s, t) \mathrm{d} W_{s}\right) \mathrm{d} t
$$

Las derivadas parciales de las integrales del lado derecho de la ecuación anterior se calculan mediante la regla de Leibniz, de tal forma que

$$
\frac{\partial}{\partial t}\left(\int_{0}^{t} \alpha(s, t) \mathrm{d} s\right) \mathrm{d} t=\left(\alpha(t, t)+\int_{0}^{t} \frac{\partial \alpha(s, t)}{\partial t} \mathrm{~d} s\right) \mathrm{d} t
$$

$\mathrm{y}$

$$
\frac{\partial}{\partial t}\left(\int_{0}^{t} \varphi(s, t) d W_{s}\right) \mathrm{d} t=\varphi(t, t) \mathrm{d} W_{t}+\left(\int_{0}^{t} \frac{\partial \varphi(s, t)}{\partial t} \mathrm{~d} W_{s}\right) \mathrm{d} t
$$

En consecuencia, la ecuación (45) puede expresarse como: 


$$
\mathrm{d} r_{t}=\left(\frac{\partial f(0, t)}{\partial t}+\alpha(t, t)+\int_{0}^{t} \frac{\partial \alpha(s, t)}{\partial t} \mathrm{~d} s+\int_{0}^{t} \frac{\partial \varphi(s, t)}{\partial t} \mathrm{~d} W_{s}\right) \mathrm{d} t+\varphi(t) \mathrm{d} W_{t}
$$

donde $\varphi(t)=\varphi(t, t)$ es la volatilidad de la tasa corta. Esta ecuación determina el comportamiento de la tasa corta. Observe que la tendencia de $r_{t}$ es la pendiente de la tasa forward inicial. Evidentemente, debido a la presencia de las integrales de la tendencia en (46), la evolución de la tasa corta no presenta la propiedad markoviana. Una vez que se ha determinado la dinámica que gobierna el comportamiento de $r_{t}$, dada en la ecuación (46), se describirá, en las secciones subsecuentes, la dinámica del precio del bono cupón cero asociado a $r_{t}$.

\subsection{Dinámica estocástica del precio del bono}

Dada la especificación exógena de la dinámica estocástica de la tasa forward instantánea, el objetivo de esta sección consiste en determinar endógenamente el precio del bono, $B(t, T)$, que sea consistente con los supuestos (41) y (42). Sea

$$
I_{t}=-\int_{t}^{T} f(t, s) \mathrm{d} s
$$

En este caso, la regla de Leibniz produce el siguiente resultado:

$$
\mathrm{d} I_{t}=-\frac{\partial}{\partial t}\left(\int_{t}^{T} f(t, s) \mathrm{d} s\right) \mathrm{d} t=-\int_{t}^{T}\left(\frac{\partial f(t, s)}{\partial t} \mathrm{~d} t\right) \mathrm{d} s+f(t, t) \mathrm{d} t
$$

La sustitución de (41) en (48) y el hecho de que $f(t, t)=r_{t}$ conducen a

$$
\begin{aligned}
\mathrm{d} I_{t} & =-\int_{t}^{T} \alpha(t, s) \mathrm{d} s \mathrm{~d} t-\int_{t}^{T} \varphi(t, s) \mathrm{d} s \mathrm{~d} W_{t}+r_{t} \mathrm{~d} t \\
& =\left(r_{t}-\int_{t}^{T} \alpha(t, s) \mathrm{d} s\right) \mathrm{d} t-\left(\int_{t}^{T} \varphi(t, s) \mathrm{d} s\right) \mathrm{d} W_{t}
\end{aligned}
$$

Si se denotan la tendencia y volatilidad de $\mathrm{d} I_{t}$, respectivamente, mediante

$$
U\left(t, T, r_{t}\right)=r_{t}-\int_{t}^{T} \alpha(t, s) \mathrm{d} s
$$

$\mathrm{y}$

$$
V(t, T)=-\int_{t}^{T} \varphi(t, s) \mathrm{d} s=-\sigma(t, T)
$$

se sigue que 


$$
\mathrm{d} I_{t}=U\left(t, T, r_{t}\right) \mathrm{d} t+V(t, T) \mathrm{d} W_{t}
$$

Note ahora que

$$
B(t, T)=G\left(I_{t}\right) \quad \text { con } \quad G\left(I_{t}\right)=\exp \left\{I_{t}\right\}
$$

En consecuencia, el lema de Itô aplicado a $G$ con respecto al proceso (50) conduce a

$$
\begin{aligned}
\mathrm{d} B & =\left(\frac{\partial G}{\partial I_{t}} U+(1 / 2) \frac{\partial^{2} G}{\partial I_{t}^{2}} V^{2}\right) \mathrm{d} t+\frac{\partial G}{\partial I_{t}} V \mathrm{~d} W_{t} \\
& =\left(G U+(1 / 2) G V^{2}\right) \mathrm{d} t+G V \mathrm{~d} W_{t} \\
& =B\left(U+(1 / 2) V^{2}\right) \mathrm{d} t+B V \mathrm{~d} W_{t}
\end{aligned}
$$

Equivalentemente,

$$
\begin{aligned}
\mathrm{d} B(t, T)= & {\left[r_{t}-\int_{t}^{T} \alpha(t, s) \mathrm{d} s+\frac{1}{2}\left(\int_{t}^{T} \varphi(t, s) \mathrm{d} s\right)^{2}\right] B(t, T) \mathrm{d} t } \\
& -\left(\int_{t}^{T} \varphi(t, s) \mathrm{d} s\right) B(t, T) \mathrm{d} W_{t} .
\end{aligned}
$$

El marco teórico de HJM, representado por las ecuaciones (41), (45) y (51), describe por completo los comportamientos de la tasa forward instantánea, de la tasa corta y del precio del bono. Es necesario ahora moverse al mundo neutral al riesgo para llevar a cabo el proceso de valuación.

\subsection{Valuación neutral al riesgo en el modelo HJM}

Considere un portafolio con dos bonos con vencimientos diferentes, $T_{1}$ y $T_{2}$. El valor del portafolio, en el tiempo $t$, con $w_{1}$ unidades del bono con vencimiento en $T_{1} \mathrm{y} \mathrm{w}_{2}$ unidades del bono con vencimiento en $T_{2}$ está dado por:

$$
\Pi_{t}=w_{1} B\left(t, T_{1}\right)+w_{2} B\left(t, T_{2}\right) .
$$

El cambio en el valor del portafolio por fluctuaciones propias del mercado satisface

$$
\begin{aligned}
\mathrm{d} \Pi_{t}= & {\left[w_{1}\left(U\left(t, T_{1}, r_{t}\right)+(1 / 2) V^{2}\left(t, T_{1}\right)\right) B\left(t, T_{1}\right)\right.} \\
& \left.+w_{2}\left(U\left(t, T_{2}, r_{t}\right)+(1 / 2) V^{2}\left(t, T_{2}\right)\right) B\left(t, T_{2}\right)\right] \mathrm{d} t \\
& +\left[w_{1} V\left(t, T_{1}\right) B\left(t, T_{1}\right)+w_{2} V\left(t, T_{2}\right) B\left(t, T_{2}\right)\right] \mathrm{d} W_{t} .
\end{aligned}
$$

Si se escogen $w_{1}=1 \mathrm{y}$ 


$$
w_{2}=-\frac{V\left(t, T_{1}\right) B\left(t, T_{1}\right)}{\mathrm{V}\left(t, T_{2}\right) B\left(t, T_{2}\right)}
$$

entonces el coeficiente del término en $\mathrm{d} W_{t}$ se anula y, consecuentemente, el portafolio se encuentra cubierto contra el riesgo de mercado. Por lo tanto,

$$
\mathrm{d} \Pi_{t}=\left(U\left(t, T_{1}, r_{t}\right)+(1 / 2) V^{2}\left(t, T_{1}\right)-\frac{U\left(t, T_{2}, r_{t}\right)+(1 / 2) V^{2}\left(t, T_{2}\right)}{V\left(t, T_{2}\right)} V\left(t, T_{1}\right)\right) B\left(t, T_{1}\right) \mathrm{d} t
$$

Si, por otro lado, existe un mercado de crédito en donde los agentes pueden prestar y pedir prestado a la tasa "spot" $r_{t}$, también llamada tasa corta o tasa instantánea (asociada, en la práctica, a la tasa de interés de plazo más pequeño disponible en el mercado), se sigue que

$$
\mathrm{d} \Pi_{t}=\Pi_{t} r_{t} \mathrm{~d} t=\left(1-\frac{V\left(t, T_{1}\right)}{V\left(t, T_{2}\right)}\right) r_{t} B\left(t, T_{1}\right) \mathrm{d} t
$$

Después de igualar (55) con (56), se obtiene

$$
\frac{U\left(t, T_{1}, r_{t}\right)+\frac{1}{2} V^{2}\left(t, T_{1}\right)-r_{t}}{V\left(t, T_{1}\right)}=\frac{U\left(t, T_{2}, r_{t}\right)+\frac{1}{2} V^{2}\left(t, T_{2}\right)-r_{t}}{V\left(t, T_{2}\right)}
$$

Los cocientes anteriores son independientes de la fecha de vencimiento. Es decir, el lado izquierdo de la igualdad en (57) sólo depende de $T_{1}$ y el derecho sólo depende de $T_{2}$. Por lo tanto, se puede escribir

$$
\lambda\left(r_{t}, t\right)=\frac{U\left(t, T, r_{t}\right)+\frac{1}{2} V^{2}(t, T)-r_{t}}{V(t, T)}
$$

La función $\lambda\left(r_{t}, t\right)$ es el premio al riesgo asociado al factor de incertidumbre $\mathrm{d} W_{t}$. En un mundo neutral al riesgo $\lambda\left(r_{t}, t\right) \equiv 0 .{ }^{6}$ En consecuencia, el supuesto de neutralidad al riesgo en el modelo HJM conduce a:

Equivalentemente,

$$
U\left(t, T, r_{t}\right)+(1 / 2) V^{2}(t, T)=r_{t}
$$

\footnotetext{
${ }^{6}$ Lo correcto es decir que existe una medida martingala equivalente.
} 


$$
r_{t}-\int_{t}^{T} \alpha(t, s) \mathrm{d} s+(1 / 2)\left(\int_{t}^{T} \varphi(t, s) \mathrm{d} s\right)^{2}=r_{t},
$$

lo cual implica

$$
\int_{t}^{T} \alpha(t, s) \mathrm{d} s=(1 / 2)\left(\int_{t}^{T} \varphi(t, s) \mathrm{d} s\right)^{2}
$$

Después de derivar la expresión anterior con respecto de $T$, se obtiene que

$$
\alpha(t, T)=\varphi(t, T) \int_{t}^{T} \varphi(t, s) \mathrm{d} s
$$

Observe que la integral de la volatilidad de la tasa forward es la volatilidad del precio del bono como en la ecuación (38). Si, con base en (28), se escribe

$$
\sigma(t, T)=\int_{t}^{T} \varphi(t, s) \mathrm{d} s=-V(t, T)
$$

entonces

$$
-\sigma(t, T)=-\frac{\alpha(t, T)}{\varphi(t, T)}=V(t, T)
$$

Por lo tanto, la ecuación del precio del bono definido en (51), bajo el supuesto de neutralidad al riesgo, se transforma en

$$
\begin{aligned}
\mathrm{d} B(t, T) & =r_{t} B(t, T) \mathrm{d} t-\left(\int_{t}^{T} \varphi(t, s) \mathrm{d} s\right) B(t, T) \mathrm{d} W_{t} \\
& =r_{t} B(t, T) \mathrm{d} t-\frac{\alpha(t, T)}{\varphi(t, T)} B(t, T) \mathrm{d} W_{t} . \\
& =r_{t} B(t, T) \mathrm{d} t-\sigma(t, T) B(t, T) \mathrm{d} W_{t} .
\end{aligned}
$$

La componente determinista de la ecuación anterior implica crecimiento exponencial en el precio del bono con tendencia igual a la tasa corta. De esta manera, la ecuación diferencial estocástica que gobierna la dinámica de la tasa forward instantánea, bajo el supuesto de neutralidad al riesgo, toma ahora la forma:

$$
\mathrm{d} f(t, T)=\left(\varphi(t, T) \int_{t}^{T} \varphi(t, s) \mathrm{d} s\right) \mathrm{d} t+\varphi(t, T) \mathrm{d} W_{t}
$$


En consecuencia, la tendencia de la tasa forward se calibra, implícitamente, en función de su volatilidad $\varphi(t, T)$.

\subsection{Representaciones alternativas de las tasas forward y corta}

Es frecuente encontrar en la literatura otras representaciones de las tasas forward y corta en la metodología HJM. Si se denota

$$
V(t, T)=-\int_{t}^{T} \varphi(t, s) \mathrm{d} s=-\sigma(t, T)
$$

se tiene, en virtud de (51) y (54), que

$$
\mathrm{d} f(t, T)=V_{T}(t, T) V(t, T) \mathrm{d} t-V_{T}(t, T) \mathrm{d} W_{t}
$$

$\mathrm{y}$

$$
r_{t}=f(0, t)+\int_{0}^{t} V_{t}(s, t) V(s, t) \mathrm{d} s-\int_{0}^{t} V_{t}(s, t) \mathrm{d} W_{s}
$$

La aplicación de la regla de Leibniz y el hecho de que $V(t, t)=0$, conducen ahora a:

$$
\mathrm{d} r_{t}=f_{t}(0, t) \mathrm{d} t+\left(\int_{0}^{t}\left[V_{t t}(s, t) V(s, t)+V_{t}(s, t)^{2}\right] \mathrm{d} s\right) \mathrm{d} t-\left(\int_{0}^{t} V_{t t}(s, t) \mathrm{d} W_{s}\right) \mathrm{d} t-V_{t}(t, t) \mathrm{d} W_{t}
$$

Por último, observe que el precio del bono satisface

$$
\mathrm{d} B(t, T)=r_{t} B(t, T) \mathrm{d} t+V(t, T) B(t, T) \mathrm{d} W_{t}
$$

equivalentemente

$$
\mathrm{d} B(t, T)=r_{t} B(t, T) \mathrm{d} t-\sigma(t, T) B(t, T) \mathrm{d} W_{t}
$$

\subsection{Un primer ejemplo de la metodología HJM}

En esta sección se ilustra la metodología HJM a través de un ejemplo sencillo. Suponga que $\varphi(t, T)=$ $\varphi_{0}=$ constante. Observe, primero, que con base en (60), se sigue que

$$
\alpha(t, T)=\varphi(t, T) \int_{t}^{T} \varphi(t, s) \mathrm{d} s=\varphi_{0}^{2}(T-t)
$$

En virtud de (63), se cumple que 
Así,

$$
\begin{gathered}
f(t, T)=f(0, T)+\varphi_{0}^{2} \int_{0}^{t}(T-s) \mathrm{d} s+\varphi_{0} W_{t} \\
=f(0, T)+\varphi_{0}^{2} t(T-(1 / 2) t)+\varphi_{0} W_{t} .
\end{gathered}
$$

$$
r_{t}=f(0, t)+(1 / 2) \varphi_{0}^{2} t^{2}+\varphi_{0} W_{t}
$$

De la misma manera, la ecuación (61) conduce a

$$
\begin{gathered}
\mathrm{d} B(t, T)=r_{t} B(t, T) \mathrm{d} t-\frac{\alpha(t, T)}{\varphi(t, T)} B(t, T) \mathrm{d} W_{t} \\
=r_{t} B(t, T) \mathrm{d} t-\varphi_{0}(T-t) B(t, T) \mathrm{d} W_{t} .
\end{gathered}
$$

Por otro lado, (52) implica que

$$
\begin{aligned}
B(t, T) & =\exp \left\{-\int_{t}^{T} f(0, s) \mathrm{d} s-\int_{t}^{T}\left[\varphi_{0}^{2} t(s-(1 / 2) t)+\varphi_{0} W_{t}\right] \mathrm{d} s\right\} \\
& =\exp \left\{\int_{0}^{t} f(0, s) \mathrm{d} s-\int_{0}^{T} f(0, s) \mathrm{d} s-\int_{t}^{T}\left[\varphi_{0}^{2} t(s-(1 / 2) t)+\varphi_{0} W_{t}\right] \mathrm{d} s\right\} \\
& =\frac{B(0, T)}{B(0, t)} e^{-\frac{1}{2} \varphi_{0}^{2} t T(T-t)-\varphi_{0}(T-t) W_{t}}
\end{aligned}
$$

De lo anterior, se observa la dependencia del precio $B(t, T)$ con una curva de rendimiento disponible en el tiempo $t=0, R(0, T)=-\ln B(0, T) / T$. Es también importante destacar que $B(t, T)$ es variable aleatoria debido a la presencia de $W_{t}$ en el término exponencial.

\subsection{Un segundo ejemplo de la metodología HJM}

La importancia de la metodología HJM se entiende mejor a través de ejemplos ilustrativos. Con este propósito en mente, se desarrolla un ejemplo más. Suponga ahora que

$$
\varphi(t, T)=\varphi_{0} e^{-\kappa(T-t)}
$$

como en el modelo de Vasicek. En virtud de la ecuación (60), se sigue que

$$
\alpha(t, T)=\varphi(t, T) \int_{t}^{T} \varphi(t, s) \mathrm{d} s=\frac{\varphi_{0}^{2}}{\kappa}\left(e^{-\kappa(T-t)}-e^{-2 \kappa(T-t)}\right)
$$

Si ahora se utiliza (53), se obtiene 


$$
\begin{aligned}
f(t, T) & =f(0, T)+\int_{0}^{t} \alpha(s, T) \mathrm{d} s+\int_{0}^{t} \varphi(s, T) \mathrm{d} W_{s} \\
& =f(0, T)+\frac{\varphi_{0}^{2}}{\kappa} \int_{0}^{t}\left(e^{-\kappa(T-s)}-e^{-2 \kappa(T-s)}\right) \mathrm{d} s+\varphi_{0} \int_{0}^{t} e^{-\kappa(T-s)} \mathrm{d} W_{s} \\
& =f(0, T)+\frac{\varphi_{0}^{2}}{2 \kappa^{2}}\left[2 e^{-\kappa T}\left(e^{\kappa t}-1\right)-e^{-2 \kappa T}-\left(e^{2 \kappa t}-1\right)\right]+\varphi_{0} \int_{0}^{t} e^{-\kappa(T-s)} \mathrm{d} W_{s}
\end{aligned}
$$

Así,

$$
r(t)=f(0, t)+\frac{\varphi_{0}^{2}}{2 \kappa^{2}}\left(1-e^{-\kappa t}\right)^{2}+\varphi_{0} \int_{0}^{t} e^{-\kappa(t-s)} \mathrm{d} W_{s}
$$

Al comparar las últimas dos ecuaciones se obtiene una expresión para la tasa forward futura en función de la tasa corta,

$$
f(t, s)=e^{-\kappa(s-t)}(r(t)-f(0, t))+f(0, s)+\frac{\varphi_{0}^{2}}{2 \kappa^{2}} e^{-\kappa(s-t)}\left(1-e^{-\kappa(s-t)}\right)\left(1-e^{-2 \kappa t}\right) .
$$

Para un valor dado de $r=r(t), f(t, s)$ es una función determinista de $s$ and $t$ que no requiere que sea actualizada, no obstante, ésta será reemplazada por la curva de la tasa forward realizada al tiempo $t$. Asimismo, la ecuación diferencial estocástica que conduce la dinámica de la tasa corta $r=r(t)$ está dada por

$$
\mathrm{d} r=\kappa(\theta(t)-r) \mathrm{d} t+\varphi_{0} \mathrm{~d} W_{t}
$$

donde

$$
\theta(t)=f(0, t)+\frac{1}{\kappa} \frac{\partial}{\partial t} f(0, t)+\frac{\varphi_{0}^{2}}{2 \kappa^{2}}\left(1-e^{-2 \kappa t}\right) .
$$

El modelo HJM supone que en $t=0, \theta(t)$ es una función determinista para toda $t \geq 0$. Sin embargo, ésta cambiará en fechas posteriores cuando se haga la revisión de precios. Lo mismo es cierto para el modelo de Hull y White y otros modelos descriptivos.

Ahora bien, la ecuación (61) conduce a

$$
\begin{aligned}
\mathrm{d} B(t, T) & =r_{t} B(t, T) \mathrm{d} t-\frac{\alpha(t, T)}{\varphi(t, T)} B(t, T) \mathrm{d} W_{t} \\
& =r_{t} B(t, T) \mathrm{d} t-\frac{\varphi_{0}}{\kappa}\left(1-e^{-\kappa(T-t)}\right) B(t, T) \mathrm{d} W_{t}
\end{aligned}
$$

Por lo tanto,

$$
B(t, T)=\exp \left\{-\int_{t}^{T}\left[f(0, s)+\frac{\varphi_{0}^{2}}{2 \kappa^{2}}\left[2 e^{-\kappa s}\left(e^{\kappa t}-1\right)-e^{-2 \kappa s}\left(e^{2 \kappa t}-1\right)\right]+\varphi_{0} \int_{0}^{t} e^{-\kappa(s-u)} \mathrm{d} W_{u}\right] \mathrm{d} s\right\}
$$




$$
\begin{aligned}
=\frac{B(0, T)}{B(0, t)} \exp & \left\{-\frac{\varphi_{0}^{2}}{\kappa^{3}}\left(e^{\kappa t}-1\right)\left(e^{\kappa t}-e^{\kappa T}\right)+\frac{\varphi_{0}^{2}}{4 \kappa^{3}}\left(e^{2 \kappa t}-1\right)\left(e^{-2 \kappa t}-e^{-2 \kappa T}\right)\right. \\
& \left.-\varphi_{0} \int_{t}^{T} e^{-\kappa s}\left(\int_{0}^{t} e^{\kappa u} \mathrm{~d} W_{u}\right) \mathrm{d} s\right\} .
\end{aligned}
$$

\subsection{Dinámica de la tasa forward con dos factores de riesgo}

Si se considera un solo factor en el modelo HJM, los bonos de diferentes plazos están perfectamente correlacionados. Esta situación se puede corregir si se incluyen otros factores de riesgo. Suponga que

$$
\mathrm{d} f(t, T)=\alpha(t, T) \mathrm{d} t+\varphi_{1}(t, T) \mathrm{d} W_{1 t}+\varphi_{2}(t, T) \mathrm{d} W_{2 t}
$$

donde $\varphi_{1}(t, T)=\varphi_{01}$ y $\varphi_{2}(t, T)=\varphi_{02} e^{-\kappa(T-t)}$. Asimismo, suponga que

$$
\operatorname{Cov}\left(\mathrm{d} W_{1 t}, \mathrm{~d} W_{2 t}\right)=0
$$

El término $\mathrm{d} W_{1 t}$ es un factor de riesgo de largo plazo, ya que traslada de manera uniforme la tasa forward a todos los vencimientos. El término $\mathrm{d} W_{2 t}$ afecta la tasa forward en vencimientos pequeños más que el factor de largo plazo. Los resultados de las dos secciones anteriores conducen a

$$
\begin{aligned}
f(t, T)=f(0, T) & +\varphi_{01}^{2} t(T-(1 / 2) t)+\varphi_{01} W_{1 t}+\frac{\varphi_{02}^{2}}{2 \kappa^{2}}+\left[2 e^{-\kappa T}\left(e^{\kappa t}-1\right)-e^{-2 \kappa T}\left(e^{2 \kappa t}-1\right)\right] \\
& +\varphi_{02} \int_{0}^{t} e^{-\kappa(T-s)} \mathrm{d} W_{2 s} .
\end{aligned}
$$

Así, $r_{t}=f(0, t)+\frac{1}{2} \varphi_{01}^{2} t^{2}+\varphi_{01} W_{1 t}+\frac{\varphi_{02}^{2}}{2 \kappa^{2}}\left(1-e^{-\kappa t}\right)^{2}+\varphi_{02} \int_{0}^{t} e^{-\kappa(t-s)} \mathrm{d} W_{2 s}$.

\subsection{Versión discreta del modelo HJM}

A continuación, se considera una versión discreta del modelo de Heath, Jarrow y Morton (1990). Se examina el proceso de tasas forward en periodos de longitud $\Delta$, en lugar del proceso de tasas forward instantáneas. Se definen $\alpha_{i, j}$ y $\varphi_{i, j}, i, j=1,2, \ldots, k$, como la tendencia y la desviación estándar, respectivamente, del proceso discretizado de la tasa forward entre los tiempos j $\Delta$ y $j \Delta+\Delta$ vista al tiempo $i \Delta$. Es decir, la versión discreta de (51) está dada por:

$$
\mathrm{d} f(t, j \Delta, j \Delta+\Delta)=\alpha_{i, j} \mathrm{~d} t+\varphi_{i, j} \mathrm{~d} W_{t}
$$


cuando $t=i \Delta$. De esta manera

$$
V_{i, j} \approx \frac{V_{i, j+1}+V_{i, j}}{2}
$$

$\mathrm{y}$

$$
\frac{\Delta V_{i, j}}{\Delta} \approx \frac{V_{i, j+1}-V_{i, j}}{\Delta}
$$

Por lo tanto se puede escribir, en virtud de (63), que

$$
\alpha_{i, j}=V_{i, j} \frac{\Delta V_{i, j}}{\Delta}=\frac{V_{i, j+1}^{2}-V_{i, j}^{2}}{2 \Delta}
$$

y

$$
\varphi_{i, j}=\frac{V_{i, j+1}-V_{i, j}}{\Delta}
$$

donde $V_{i, j}$ es el valor de $V(t, T)$ cuando $t=i \Delta$ y $T=j \Delta$. Ahora bien, dado que $V_{i, i}=0$, se sigue de (59) que

$$
\begin{gathered}
\sum_{j=1}^{k} \alpha_{i, j} \Delta=\frac{1}{2}\left(\sum_{j=1}^{k} \varphi_{i, j} \Delta\right)^{2} \text { ó } \\
\sum_{j=1}^{k} \alpha_{i, j}=\frac{1}{2} \Delta\left(\sum_{j=1}^{k} \varphi_{i, j}\right)^{2} .
\end{gathered}
$$

\subsection{Simulación Monte Carlo de HJM}

El método de simulación Monte Carlo puede utilizarse para estimar el modelo HJM ya que $f(t, T)$ y $B(t, T)$ son variable aleatorias. El periodo de tiempo sobre el cual la simulación se lleva a cabo está dividido en $n$ subintervalos de la misma longitud, $\Delta$. De esta manera, la versión discreta de (51) consiste en

$$
f_{i+1, j}-f_{i, j}=\alpha_{i, j} \Delta+\varphi_{i, j} \varepsilon \sqrt{\Delta}
$$

donde $f_{i, j}$ denota a $f(i \Delta, j \Delta, j \Delta+\Delta)$, esto es, $f_{i, j}$ es la tasa forward entre los periodos $j \Delta$ y $(j+1) \Delta$ vista al tiempo $i \Delta$. Se supone que la variable aleatoria $\varepsilon$ tiene distribución normal estándar. Los valores $\alpha_{i, j}$ pueden calcularse a partir de los valores de $\varphi_{i, j}$ utilizando (66). Asimismo, al tiempo $i \Delta$, se almacenan los precios de los bonos que se tienen en el vencimiento $j \Delta$ para $i+1 \leq j \leq n$. Por otro lado, la ecuación (65) se transforma en

$$
\frac{B_{i+1, j}-B_{i, j}}{B_{i, j}}=\left(\frac{1-B_{i, j}}{B_{i, j}}\right)+V_{i, j} \varepsilon \sqrt{\Delta}
$$




$$
B_{i+1, j}=B_{i, j}\left(\frac{1}{B_{i, j}}+V_{i, j} \varepsilon \sqrt{\Delta}\right)
$$

donde $B_{i, j}$ es el precio al tiempo $i \Delta$ de un bono con vencimiento al tiempo $j \Delta$. Posteriormente se calcula $R(t, T)=-\ln B(t, T) /(T-t)$.

\section{Propuestas de investigación futura}

Con base en el desarrollo de las secciones anteriores se presentan a continuación algunas de las áreas que ofrecen oportunidades para realizar investigación; la lista no pretende ser exhaustiva,

- Estructuras de plazo con múltiples factores y varias fuentes de incertidumbre modeladas con procesos de Lévy combinados con procesos de Cox y modulados por cadenas de Markov.

- Finanzas cuánticas y estructuras de plazos

- Modelos estocásticos de equilibrio macroeconómico que expliquen el comportamiento de la tasa de interés abandonando el supuesto de normalidad.

- Efectos de tasas de interés negativas en fondos de pensiones.

- Administración del riesgo de tasas de interés de microcréditos, con múltiples factores, en el entorno de la pandemia COVID-19.

- Desarrollo de modelos macroeconómicos estocásticos con sector salud y su vulnerabilidad a eventos extremos (catástrofes, pandemias -COVID-19-, etc.) que expliquen el comportamiento futuro de las estructuras de plazos.

- Efectos de la incertidumbre en la política económica en las estructuras de plazos.

- Comportamiento de agentes financieros (finanzas conductuales) en mercados de deuda e inteligencia artificial.

- Ciencia de datos aplicada al mercado de deuda

- Efectos de la pandemia COVID-19 en los mercados de deuda y su impacto en las estructuras de plazos.

\section{Conclusiones}

En el transcurso de este trabajo se realizó una revisión de las tendencias y perspectivas de las finanzas matemáticas. También se realizó un seguimiento detallado de la evolución de la teoría de la estructura de plazos y de los modelos que se han propuesto en la literatura. Las propuestas de investigación enumeran áreas que ofrecen oportunidades para el trabajo futuro. 


\section{Referencias}

[1] Adams, K. J. and Van Deventer, D. R. (1994). Fitting Yield Curves and Forward Rate Curves With Maximum Smoothness. Journal of Fixed Income, 4(1), 52-62

[2] Black F. and P. Karasinski (1991) Bond and Option Pricing When Short Rates Are Lognormal, Financial Analysts Journal 47(4), 52-59.

[3] Brennan, M. J. and E. S. Schwartz (1979). A Continuous Time Approach to the Pricing of Bonds. Journal of Banking and Finance, 3(2), 133-155.

[4] Cox, J. C., J. E. Ingersoll, and S. A. Ross (1985). A Theory of the Term Structure of Interest Rates. Econometrica, 5(2), 363-384.

[5] Duffie, D and R. Kan (1996). A Yield-Factor Model of Interest Rates. Mathematical Finance, 6(4), 379406.

[6] Harrison, J. M. and D. M. Kreps (1979). Martingales and Arbitrage in Multiperiod Securities Markets. Journal of Economic Theory, 20(3), 381-408.

[7] Heath, D., R. Jarrow, and A. Morton (1990). Bond Pricing and the Term Structure of Interest Rates: A Discrete Time Approximation. Journal of Financial and Quantitative Analysis, 25(4), 419-440.

[8] Heath, D., R. Jarrow, and A. Morton (1992). Bond Pricing and the Term Structure of Interest Rates: A New Methodology for Contingent Claims Valuation. Econometrica, 60(1), 77-105.

[9] Ho, T. and S. Lee (1986). Term Structure Movements and Pricing Interest Rate Contingent Claims. Journal of Finance, 41(5), 1129-1142.

[10] Hull, J. and A. White (1990). Pricing Interest Rate Derivatives Securities. Review of Financial Studies, 3(4), 573-592.

[11] Langetieg, T. C. (1980). A Multivariate Model of the Term Structure. Journal of Finance, 35(1), 71-97.

[12] Longstaff F. A. and E. S. Schwartz (1992). Interest Rate Volatility and the Term Structure: A Two Factor General Equilibrium Model. Journal of Finance, 47(4), 1259-1282.

[13] Longstaff, F. A. (1989). A Nonlinear General Equilibrium Model of the Term Structure of Interest Rates. Journal of Financial Economics, 23(2), 195-224.

[14] Macaulay, F. R. (1938), Some Theoretical Problems Suggested by the Movements of Interest-Rates, Bond Yields and Stock Prices in the U.S. Since 1856. National Bureau of Economic Research, New York.

[15] McCulloch, J. H. (1971). Measuring the Term Structure of Interest Rates. Journal of Business, 1971, 44(1), 19-31.

[16] Nelson, C. R. and A. F. Siegel (1987). Parsimonious Modeling of Yield Curves. Journal of Business, 60 (4), 473-489.

[17] Vasicek, A. O. (2020). Interpolating the Yield Curve. Journal of Fixed Income, 30(2), 76-85.

[18] Vasicek, A. O. and H. G. Fong (1982). Term Structure Modeling Using Exponential Splines. Journal of Finance, 37(2), 339-348.

[19] Vasicek, A. O. (1977). An Equilibrium Characterization of the Term Structure. Journal of Financial Economics, 5(2), 177-188.

[20] Vasicek, A. O. (2015). Finance, Economics, and Mathematics (2015). John Wiley \& Sons, Inc., Hoboken, New Jersey.

[21] Venegas-Martínez, F. y B. González-Aréchiga (2002). Cobertura de tasas de interés con futuros del mercado mexicano de derivados: un modelo estocástico de duración y convexidad. El Trimestre Económico, 59(274), 227-250.

[22] Venegas-Martínez, F. (2002). Cobertura de flujos financieros con instrumentos de renta fija. Estudios Económicos, 17(2), 171-192. 
[23] Venegas-Martínez, F. (2003). Inmunización de flujos financieros de tesorerías con bonos cupón cero: un análisis de duración y convexidad con el modelo de Heath, Jarrow y Morton. Momento Económico, 129-130, 3-17.

[24] Venegas-Martínez, F. (2008). Riesgos financieros y económicos: Productos derivados y decisiones económicas bajo incertidumbre, 2da. Edición. Cengage Learning, México. 